ARTICLE OPEN

\title{
Energy-participation quantization of Josephson circuits
}

\author{
Zlatko K. Minev $\mathbb{D}^{1,4 凶}$, Zaki Leghtas ${ }^{1,2}$, Shantanu O. Mundhada ${ }^{1,5}$, Lysander Christakis ${ }^{1,6}$, loan M. Pop $\mathbb{D}^{1,3}$ and Michel H. Devoret $\mathbb{D}^{1}$
}

Superconducting microwave circuits incorporating nonlinear devices, such as Josephson junctions, are a leading platform for emerging quantum technologies. Increasing circuit complexity further requires efficient methods for the calculation and optimization of the spectrum, nonlinear interactions, and dissipation in multi-mode distributed quantum circuits. Here we present a method based on the energy-participation ratio (EPR) of a dissipative or nonlinear element in an electromagnetic mode. The EPR, a number between zero and one, quantifies how much of the mode energy is stored in each element. The EPRs obey universal constraints and are calculated from one electromagnetic-eigenmode simulation. They lead directly to the system quantum Hamiltonian and dissipative parameters. The method provides an intuitive and simple-to-use tool to quantize multi-junction circuits. We experimentally tested this method on a variety of Josephson circuits and demonstrated agreement within several percents for nonlinear couplings and modal Hamiltonian parameters, spanning five orders of magnitude in energy, across a dozen samples.

npj Quantum Information (2021)7:131 ; https://doi.org/10.1038/s41534-021-00461-8

\section{INTRODUCTION}

Quantum information processing based on the control of microwave electromagnetic fields in Josephson circuits is a promising platform for both fundamental physics experiments and emerging quantum technologies ${ }^{1-3}$. Key to the success of this platform is the ability to quantitatively model the distributed quantized electromagnetic modes of the system, their nonlinear interactions, and their dissipation (see Fig. 1). This challenge is the subject of intensifying interest ${ }^{4-26}$, as experimental architectures $^{27-36}$ and nonlinear devices ${ }^{37-44}$ scale in both complexity and diversity.

In this paper, we introduce a circuit quantization method based on the concept of the energy-participation ratio (EPR). We reduce the quantization problem to answering the simple question: what fraction of the energy of mode $m$ is stored in element $j$ ? This leads to a constrained number between zero and one, the EPR, denoted $p_{m j}{ }^{45}$. This ratio is the key quantity that bridges classical and quantum circuit analysis; we show that it plays the primary role in the construction of the system many-body Hamiltonian. Furthermore, dissipation in the system is treated on equal footing by calculating the EPR $p_{m /}$ of lossy element $l$ in mode $m$.

The EPR method deviates from previous black-box quantization work ${ }^{4,6,7}$, which uses the impedance-response matrix, denoted $Z_{j j^{\prime}}(\omega)$, where $j$ and $j^{\prime}$ are index ports associated with nonlinear elements. For all pairs of ports, the complex function $Z_{j j^{\prime}}(\omega)$ is calculated from a finite-element (FE) driven simulation in the vicinity of the eigenfrequency of every mode. Our method replaces these steps with a more economical FE eigenmode simulation, from which one extracts the energy participations $p_{m l}$ and $p_{m j}$, needed to fully characterize both the dissipative and Hamiltonian properties of the circuit.

To test the method, we compared EPR calculations of circuit parameters to experimentally measured ones for 8 superconducting devices designed with the EPR method, comprising a total of 15 qubits, 8 readout and storage resonator modes, and 1 waveguide system. The results demonstrate agreement for
Hamiltonian parameters spanning over five orders of magnitude in energy. Resonance frequencies were calculated to $1 \%$ accuracy, large nonlinear interactions, such as anharmonicities and crossKerr frequencies, to $5 \%$, and small, nonlinear interactions to $10 \%$. This level of accuracy is sufficient for most current quantum information experiments.

\section{RESULTS AND DISCUSSION}

\section{To quantize a simple circuit: qubit coupled to a cavity}

In this section, we introduce the EPR method of quantum circuit design on a modest, yet informative, example: a transmon qubit coupled to a cavity mode (see Fig. 2). The transmon ${ }^{46}$ consists of a Josephson junction shunted by a capacitance. It is embedded in the cavity, which we will consider as a black-box distributed structure. The Hamiltonian of this system $\hat{H}_{\text {full }}$ can be conceptually separated into two contributions (see Supplementary Section A2),

$\hat{H}_{\text {full }}=\hat{H}_{\text {lin }}+\hat{H}_{\mathrm{nl}}$,

where $\hat{H}_{\text {lin }}$ consists of all terms associated with the linear response of the junction and the resonator structure, and $\hat{H}_{\mathrm{nl}}$ consists of terms associated with the nonlinear response of the junction. Restricting our attention to the cavity and qubit modes of the otherwise black-box structure, the analytical form of the Hamiltonian follows from standard circuit quantization ${ }^{47,48}$ (see Supplementary Information):

$$
\begin{aligned}
& \hat{H}_{\text {lin }}=\hbar \omega_{c} \hat{a}_{c}^{\dagger} \hat{a}_{c}+\hbar \omega_{q} \hat{a}_{q}^{\dagger} \hat{a}_{q}, \\
& \hat{H}_{\mathrm{nl}}=-E_{J}\left[\cos \left(\hat{\varphi}_{J}\right)+\hat{\varphi}_{J}^{2} / 2\right], \\
& \hat{\varphi}_{J}=\varphi_{q}\left(\hat{a}_{q}+\hat{a}_{q}^{\dagger}\right)+\varphi_{c}\left(\hat{a}_{c}+\hat{a}_{c}^{\dagger}\right),
\end{aligned}
$$

where $\omega_{c}$ and $\omega_{q}$ are the angular frequencies of the cavity and qubit eigenmodes defined associated with $\hat{H}_{\text {lin }}$, respectively, and where $\hat{a}_{c}$ and $\hat{a}_{q}$ are their annihilation operators, respectively. The Josephson energy $E_{J}$ can be computed from the

\footnotetext{
${ }^{1}$ Department of Applied Physics, Yale University, New Haven, CT, USA. ${ }^{2}$ Centre Automatique et Systèmes, Mines-ParisTech, PSL Research University, Paris, France. ${ }^{3}$ IQMT, Karlsruhe Institute of Technology, Eggenstein-Leopoldshafen, Germany. ${ }^{4}$ Present address: IBM T.J. Watson Research Center, Yorktown Heights, NY, USA. ${ }^{5}$ Present address: Quantum Circuit Incorporated (QCI), New Haven, CT, USA. ${ }^{6}$ Present address: Department of Physics, Princeton University, Princeton, NJ, USA. ${ }^{\circledR}$ email: zlatko.minev@aya.yale.edu
} 


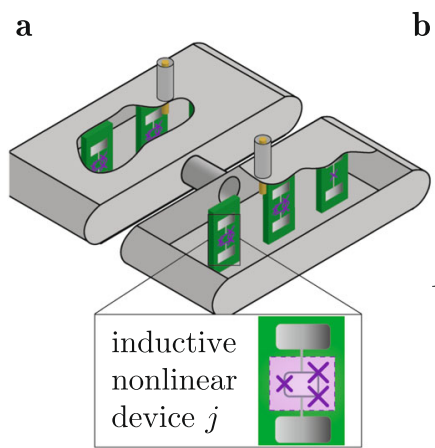

b

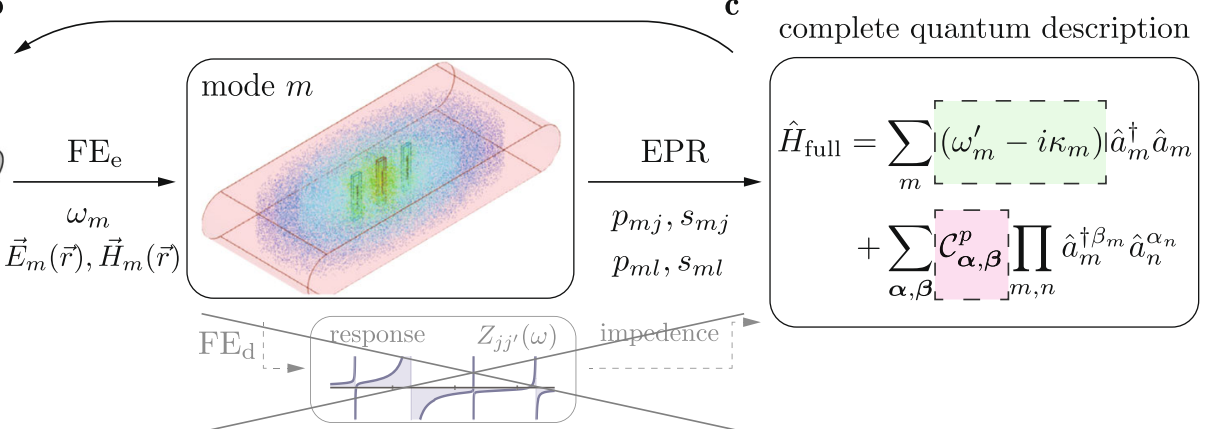

Fig. 1 Conceptual overview. a Illustration of the physical model of an example quantum device, which comprises two three-dimensional (3D) cavities (gray enclosures), each housing several qubit chips (green boxes). A close-up view of one of the chips is depicted in the inset. The dotted box in the center of the chip schematically outlines a nonlinear inductive sub-circuit, referred to as a Josephson dipole. $\mathbf{b}$ Results of a finite-element eigenmode analysis ( $\mathrm{FE}_{\mathrm{e}}$ ) of the Josephson circuit linearized about its equilibrium. The $m$ th mode eigenfrequency and electric and magnetic fields are $\omega_{m}, \vec{E}_{m}(\vec{r})$, and $\vec{H}_{m}(\vec{r})$, respectively, where $r$ denotes spatial position. Center inset: $\left|\vec{E}_{m}\right|$ profile (red: high; blue: low) for the fundamental mode of one of the 3D cavities. Additional FE driven simulations $\left(\mathrm{FE}_{\mathrm{d}}\right)$ are unnecessary; i.e., the impedance matrix $Z_{j j^{\prime}}(\omega)$ is not calculated. c The Hamiltonian $\hat{H}_{\text {full, }}$ which includes nonlinear interactions to arbitrary order (see "Results"), is computed directly from the eigenanalysis via the EPRs $p_{m j}$ and EPR signs $s_{m j}= \pm 1$ of the junctions, $j$. Dissipative contributions due to a lossy element $/$ are similarly computed from the loss EPRs $p_{m l i}$ for linear dissipation, and the EPR signs $s_{m /}$ are unnecessary. Direct extraction of Hamiltonian and dissipative parameters from eigensolutions is unique to the EPR method. The geometry of the classical model is modified in an iterative search for the desired dissipative and Hamiltonian parameters (left-pointing arrow).
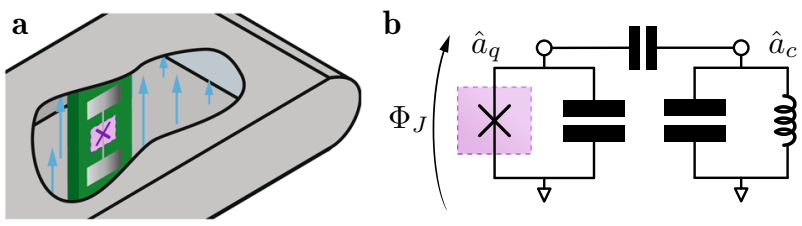

Fig. 2 Quantizing a simple circuit. a Illustration of a 3D cavity enclosing a transmon qubit chip. The cross symbol marks the location of a Josephson junction. Vertical blue arrows depict the electric field $\vec{E}_{m}(\vec{r})$ of the fundamental cavity mode, $\mathrm{TE}_{101}$. b Equivalent two-mode lumped-element representation of the distributed circuit. Operators $\hat{a}_{q}$ and $\hat{a}_{c}$ denote the qubit and cavity mode operators, respectively.

Ambegaokar-Baratoff formula adapted to the measured roomtemperature resistance of the junction ${ }^{49}$. The junction-reduced

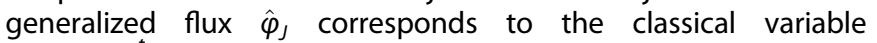
$\varphi_{J}(t):=\int_{-\infty}^{t} v_{J}(\tau) \mathrm{d} \tau / \phi_{0}$, where $v_{J}(\tau)$ is the instantaneous voltage across the junction 47,48 , and $\phi_{0}:=\hbar / 2 e$ is the reduced flux quantum. The junction flux operator [Eq. (4)] is a linear, realvalued, and non-negative combination of the mode operators (see Supplementary Section A5), and in its expression, $\varphi_{c}$ and $\varphi_{q}$ are the quantum zero-point fluctuations of junction flux in the cavity and qubit mode, respectively. It is worth stating that the linear coupling between the cavity and qubit, commonly denoted $g^{46}$, is fully factored in our analysis and is implicitly handled in the extraction of the operators from the electromagnetic simulation.

Our principal aim is to determine the unknown quantities: $\omega_{c}, \omega_{q}$, $\varphi_{q}$, and $\varphi_{c}$. As we will show, we extract and compute these quantities from an eigenanalysis of the classical distributed circuit corresponding to $\hat{H}_{\text {lin }}$. This includes the qubit-cavity layout, materials, electromagnetic boundary conditions, and a model of the junction as a lumped-element, linear inductor. The eigensolver returns the requested set of eigenmodes and their frequencies, quality factors, and field solutions. By running the eigensolver in the frequency range of interest, we obtain the hybridized cavity and qubit modes, whose eigenfrequencies $\omega_{c}$ and $\omega_{q}$ fully determine $\hat{H}_{\text {lin }}$ (see Supplementary Section C for FE methodology).

To determine $\hat{H}_{\mathrm{nl}}$, we need the quantum zero-point fluctuations $\varphi_{q}$ and $\varphi_{c}$, which are calculated from the participation of the junction in the eigenfield solutions. The participation $p_{m}$ of the junction in mode $m \in\{c, q\}$ is defined to be the fraction of inductive energy stored in the junction relative to the total inductive energy stored in the entire circuit,

$p_{m}:=\frac{\text { Inductive energy stored in the junction }}{\text { Total inductive energy stored in mode } m}$,

evaluated when only mode $\underline{m}$ is excited. Thus, $p_{m}$ can be computed from the electric $\vec{E}_{m}(\vec{r})$ and magnetic $\vec{H}_{m}(\vec{r})$ eigenfields as detailed in Supplementary Section $\mathrm{C}_{2} ; \vec{r}$ denotes spatial position. In the quantum setting, Eq. (5) links $p_{m}, \hat{\varphi}_{J}$, and the state of the circuit,

$p_{m}=\frac{\left\langle\psi_{m}\left|\frac{1}{2} E_{J} \hat{\varphi}_{J}^{2}\right| \psi_{m}\right\rangle}{\left\langle\psi_{m}\left|\frac{1}{2} \hat{H}_{\text {lin }}\right| \psi_{m}\right\rangle}$,

where $\left|\psi_{m}\right\rangle$ denotes a coherent state or a Fock excitation of mode $m$. Note that normal ordering must be used in Eq. (6); this correct treatment of vacuum fluctuations is detailed in Supplementary Section A6. Simplifying Eq. (6), one expresses the variance of the quantum zero-point fluctuations $\varphi_{c}$ and $\varphi_{q}$ as a function of the classical energy participations $p_{m}$,

$\varphi_{c}^{2}=p_{c} \frac{\hbar \omega_{c}}{2 E_{J}}$ and $\varphi_{q}^{2}=p_{q} \frac{\hbar \omega_{q}}{2 E_{\jmath}}$,

which completely determines $\hat{H}_{\mathrm{nl}}$ and thus completes the description of the system Hamiltonian $\hat{H}_{\text {full. }}$. Here $\varphi_{c}$ and $\varphi_{q}$ can be taken as positive numbers. As we will see in the next section, in the presence of multiple junctions, this is not always true.

Designing experiments with the EPR requires one to further extract from $\hat{H}_{\text {full }}$ the transition frequencies and nonlinear couplings between modes. Depending on the case, this can be done approximately or exactly using numerical or analytical techniques ${ }^{20}$. This task is easily achieved if $\hat{H}_{\mathrm{nl}}$ is a perturbation to $\hat{H}_{\text {lin }}{ }^{46}$. In this limit, $\hat{H}_{\text {full }}$ for our qubit-cavity example can be approximated by the effective, excitation-number-conserving Hamiltonian, see Supplementary Section B1,

$$
\begin{aligned}
\hat{H}_{\text {eff }}= & \left(\omega_{q}-\Delta_{q}\right) \hat{n}_{q}+\left(\omega_{c}-\Delta_{c}\right) \hat{n}_{c}-X_{q c} \hat{n}_{q} \hat{n}_{c} \\
& -\frac{1}{2} a_{q} \hat{n}_{q}\left(\hat{n}_{q}-\hat{1}\right)-\frac{1}{2} a_{c} \hat{n}_{c}\left(\hat{n}_{c}-\hat{1}\right),
\end{aligned}
$$

where $\hat{n}_{q}=\hat{a}_{q}^{\dagger} \hat{a}_{q}$ and $\hat{n}_{c}=\hat{a}_{c}^{\dagger} \hat{a}_{c}$ denote the qubit and cavity excitation-number operators, respectively, $\Delta_{q}$ denotes the "Lamb shift" of the qubit frequency due to the dressing of this nonlinear 


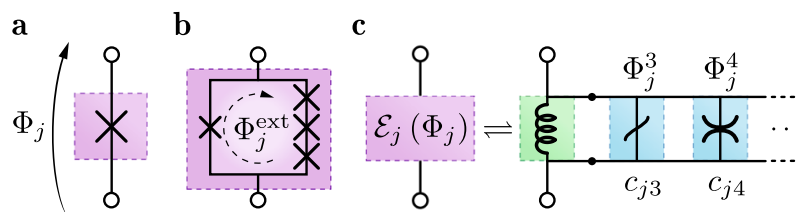

d

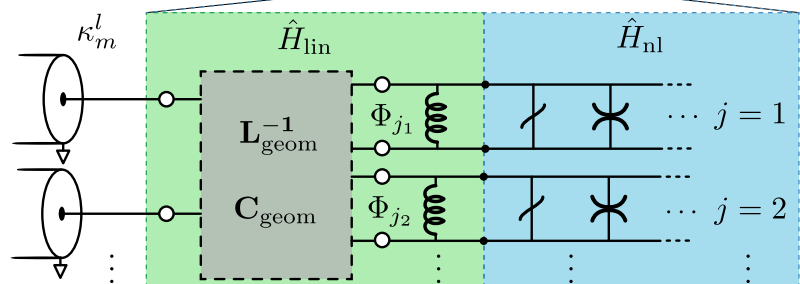

Fig. 3 Schematic representation of the Josephson circuit and its nonlinear elements. a A simple example of a Josephson dipole-a Josephson tunnel junction. b An example of a composite junction, comprising four Josephson junctions in a ring, frustrated by an external magnetic flux $\Phi_{j}^{\text {ext }}$ threading the loop. c Conceptual decomposition of a general Josephson dipole, denoted $j$. For convenience, its potential energy function $\mathcal{E}_{j}\left(\Phi_{j} ; \Phi_{j}^{\text {ext }}\right)$ can be Taylor expanded in a sum of nonlinear inductive contributions of increasing order $\Phi_{j}^{p}$, with relative amplitude $c_{j p}$, where $p$ denotes the index in the series. The energy function can be subjected to external bias parameters $\Phi_{j}^{\text {ext }}$, such as flux or voltages. d Schematic diagram of a general Josephson circuit conceptually resolved into a purely dissipative (left, $\kappa_{m}^{\prime}$ ), linear (middle, $\hat{H}_{\text {lin }}$ ), and nonlinear (right, $\hat{H}_{\text {nl }}$ ) constitutions.

mode by quantum fluctuations of the fields, $a_{q}\left(a_{c}\right)$ is the qubit (cavity) anharmonicity, and $X_{q c}$ is the qubit-cavity dispersive shift (cross-Kerr coupling). The Hamiltonian parameters can be calculated directly from the EPR, see Supplementary Section B2,

$a_{q}=\frac{1}{2} X_{q q}=p_{q}^{2} \frac{\hbar \omega_{q}^{2}}{8 E_{\jmath}}$,

$a_{c}=\frac{1}{2} X_{c c}=p_{c}^{2} \frac{\hbar \omega_{c}^{2}}{8 E_{J}}$

$X_{q c}=p_{q} p_{c} \frac{\hbar \omega_{q} \omega_{c}}{4 E_{J}}$.

Experimentally, the qubit Lamb shift can be obtained as $\Delta_{q}=a_{q}-$ $X_{a c} / 2$. Since a single EPR $p_{m}$ determines the nonlinear interaction for each mode, the parameters $X_{q c}$ and $a_{q}$ are interdependent,

$X_{q c}=\sqrt{X_{q q} X_{c c}}=2 \sqrt{a_{q} a_{c}}$.

As shown in Supplementary Section A7, the EPRs $p_{c}$ and $p_{q}$ obey the constraints

$0 \leq p_{q}, p_{c} \leq 1$ and $p_{q}+p_{c}=1$.

These relations together with Eqs. (9) and (12) are useful to budget the dilution of the nonlinearity of the junction (see Supplementary Section B2) and to provide insight on the limits of accessible parameters (see "Methods"). Further, Eq. (13) is used to validate the convergence of the FE simulation.

\section{Quantizing the general Josephson system}

The simple results obtained in the preceding section will now be generalized to arbitrary nonlinear devices enclosed in a black-box, distributed, electromagnetic structure. While such structures are frequently classified as planar ${ }^{27,50-52}$ (two-dimensional), quasiplanar $^{28,29,32,53}$ (2.5-dimensional (2.5D)), or three-dimensional ${ }^{30,54-56}$
(3D), we will treat all classes on equal footing. The electromagnetic structure is assumed to be linear in the absence of the enclosed nonlinear devices. For simplicity of discussion, we can consider these devices to be inductive and lumped; distributed nonlinear devices, such as kinetic-inductance transmission lines ${ }^{57-60}$, can be thought of as a series of lumped ones.

The simplest nonlinear device comprises a single element, such as a Josephson tunnel junction [see Fig. 3a], an atomic-point contact $^{61,62}$, a nanobridge ${ }^{38,63}$, a semiconducting nanowire ${ }^{40,41,64-}$ 67 , or another hybrid structure ${ }^{68}$. A multi-element device, such as a SQUID $^{69,70}$, a SNAIL ${ }^{71}$ [see Fig. 3b], a superinductance ${ }^{72-74}$, or a junction array ${ }^{5,75-80}$ refers to a subcircuit composed of purely inductive lumped elements. This subcircuit can also be subjected to external controls, such as voltage or flux biases.

The general nonlinear device that we now consider, referred to as a Josephson dipole, is any lumped, purely inductive, nonlinear subcircuit with two terminals. The key characteristic of the Josephson dipole is that it possesses a characteristic energy function, which encapsulates all details of its constitution. For example, the two-terminal nonlinear device known as the symmetric SQUID ${ }^{70}$ is described by the energy function $\mathcal{E}_{j}\left(\Phi_{j} ; \Phi_{j}^{\text {ext }}\right)=-E_{j}\left(\Phi_{j}^{\text {ext }}\right) \cos \left(\Phi_{j} / \phi_{0}\right)$, where $\Phi_{j}$ is the generalized flux across the device terminals ${ }^{47,48}, E_{j}$ is the effective Josephson energy, $\Phi_{j}^{\text {ext }}$ is the external flux bias, and the subscript $j$ denotes the $j$ th Josephson dipole in the circuit. The flux $\Phi_{j}$ is defined as the deviation away from the value in equilibrium, as discussed below. To ease the notation, parameters such as $\Phi^{\text {ext }}$ will be implicit hereafter. Similarly to the example of the single-junction transmon, the energy of a Josephson dipole can be separated in two parts. One part $\mathcal{E}_{j}^{\text {lin }}$ accounts for the linear response of the dipole, while the other $\mathcal{E}_{j}^{\text {nl }}$ accounts for the nonlinear response,

$\mathcal{E}_{j}\left(\Phi_{j}\right)=\mathcal{E}_{j}^{\mathrm{lin}}\left(\Phi_{j}\right)+\mathcal{E}_{j}^{\mathrm{nl}}\left(\Phi_{j}\right)$

where

$\mathcal{E}_{j}^{\operatorname{lin}}\left(\Phi_{j}\right):=\frac{1}{2} E_{j}\left(\frac{\Phi_{j}}{\phi_{0}}\right)^{2}$,

and where the constant $E_{j}$ sets the scale of the junction energy. This energy scale can be represented by the linear inductance $L_{j}:=\phi_{0} / E_{j}$ presented by the Josephson dipole when submitted to a small excitation about its equilibrium.

Frustrated equilibrium. External biases can set up persistent currents in the circuit. These can alter the static (direct current) equilibrium of the Josephson system. For example, frustrating a superconducting ring with a magnetic flux sets up a persistent circulating current in the ring. For a Josephson dipole in such a loop, the definition of the flux $\Phi_{j}$ will differ in Eqs. (14) and (15) as a function of the equilibrium. Due to this adjustment, terms linear in $\Phi_{j}$ are absent from Eq. (15) by construction. Supplementary Sections A8 and A9 discuss these equilibrium considerations in detail.

Quantum Hamiltonian. Having conceptually carved out nonlinear contributions from the system Hamiltonian $\hat{H}_{\text {full }}$ and collected them in the set of $\mathcal{E}_{j}^{\text {nl }}$ functions, we define the linearized Josephson circuit to correspond to everything left over in the system. This linear circuit consists of the electromagnetic circuit external to the Josephson dipoles, combined with their linear inductances $L_{j}$. We will use the eigenmodes of the linearized circuit to explicitly construct $\hat{H}_{\text {full. }}$. The eigenmode frequencies and field distributions are readily obtained using a conventional FE solver (see Supplementary Section C). The Hamiltonian of the linearized Josephson circuit can thus be expressed as (see Supplementary Section A5)

$\hat{H}_{\text {lin }}=\sum_{m=1}^{M} \hbar \omega_{m} \hat{a}_{m}^{\dagger} \hat{a}_{m}$, 
where $M$ is the number of modes addressed by the numerical simulation, $\omega_{m}$ is the solution eigenfrequency of mode $m$, and $\hat{a}_{m}$ the corresponding mode amplitude (annihilation operator), defined by the mode eigenvector. We emphasize that the frequencies $\omega_{m}$ will be significantly perturbed by the Lamb shifts $\Delta_{m}$ and should be seen as an intermediate parameter entering in the calculation of the rest of the nonlinear Hamiltonian,

$$
\begin{aligned}
\hat{H}_{\mathrm{nl}} & =\sum_{j=1}^{J} \mathcal{E}_{j}^{\mathrm{nl}}=\sum_{j=1}^{J} E_{j}\left(c_{j 3} \hat{\varphi}_{j}^{3}+c_{j 4} \hat{\varphi}_{j}^{4}+\cdots\right) \\
& =\sum_{j=1}^{J} E_{j} \sum_{p=3}^{\infty} c_{j p} \hat{\varphi}_{j}^{p}, \\
\hat{\varphi}_{j} & =\sum_{m=1}^{M} \varphi_{m j}\left(\hat{a}_{m}^{\dagger}+\hat{a}_{m}\right),
\end{aligned}
$$

where $J$ is the total number of junctions and $\hat{\varphi}_{j}:=\hat{\Phi}_{j} / \phi_{0}$. In Eq. (17), we have introduced a Taylor expansion of $\mathcal{E}_{j}^{\text {nl }}$, where the energy $E_{j}$ and expansion coefficients $c_{j p}$ are known from the fabrication of the Josephson circuit, see Fig. 3c. For example, for a Josephson junction, the constant $E_{j}$ is just the Josephson energy, while $c_{j p}$ are the coefficients of the cosine expansion; i.e., $c_{j p}$ is 0 for odd $p$ and $(-1)^{p / 2+1} / p$ ! for even $p$. The expansion is helpful for analytics but does not need to be used in the numerical analysis of $\hat{H}_{\mathrm{n}}$, see Supplementary Section A.

The Hamiltonian $\hat{H}_{\text {full }}$ is specified since the operators $\hat{\varphi}_{j}$ are expressed in terms of the mode amplitudes as a linear combination (see Supplementary Section A5). Here, $\varphi_{m j}$ are the dimensionless, real-valued, quantum zero-point fluctuations of the reduced flux of junction $j$ in mode $m$. Determination of $\hat{H}_{\text {full }}$ is now reduced to computing $\varphi_{m j}$. We achieve this by employing a generalization of the EPR.

The EPR $p_{m j}$ of junction $j$ in eigenmode $m$ is defined to be the fraction of inductive energy stored in the junction when only that mode is excited,

$$
\begin{aligned}
p_{m j}: & :=\frac{\text { Inductive energy stored in junction } j}{\text { Inductive energy stored in mode } m} \\
& =\frac{\left\langle\psi_{m}\left|\frac{1}{2} E_{j} \hat{\varphi}_{j}^{2}\right| \psi_{m}\right\rangle}{\left\langle\psi_{m}\left|\frac{1}{2} \hat{H}_{\text {lin }}\right| \psi_{m}\right\rangle}
\end{aligned}
$$

which is a straightforward extension of Eq. (5), and is similarly computed using normal ordering (see Supplementary Section A6). The EPR $p_{m j}$ is computed from the eigenfield solutions $\vec{E}_{m}(\vec{r})$ and $\vec{H}_{m}(\vec{r})$ as explained in Supplementary Section C3. It is a bounded, non-negative, real number, $0 \leq p_{m j} \leq 1$. A zero EPR $p_{m j}=$ 0 means that junction $j$ is not excited in mode $m$. A unity EPR $p_{m j}$ $=1$ means that junction $j$ is the only inductive element excited in the mode.

From the EPR $p_{m j}$, one directly computes the variance of the quantum zero-point fluctuations,

$\varphi_{m j}^{2}=p_{m j} \frac{\hbar \omega_{m}}{2 E_{j}}$

Equation (21) constitutes the bridge between the classical solution of the linearized Josephson circuit and the quantum Hamiltonian $\hat{H}_{\text {full }}$ of the full Josephson system, up to the sign of $\varphi_{m j}$.

Universal EPR properties: The quantum fluctuations $\varphi_{m j}$ are not independent of each other, since the EPRs are submitted to three types of universal constraints-valid regardless of the circuit topology and nature of the Josephson dipoles. These are of practical importance, as they are useful guides in evaluating the performance of possible designs and assessing their limitations. As shown in Supplementary Section A7, the EPRs obey one sum rule per junction $j$ and one set of inequalities per mode $m$,

$$
\sum_{m=1}^{M} p_{m j}=1 \text { and } 0 \leq \sum_{j=1}^{J} p_{m j} \leq 1 .
$$

The total EPR of a Josephson dipole is a quantity that is independent of the number of modes-it is precisely unity for all circuits in which the dipole is embedded. It can only be diluted among the modes. On the other hand, a given mode can accept at most a total EPR of unity from all the dipoles. In practice, this sum rule can be fully exploited only if the bound $M$ reaches the total number of relevant modes of the system.

The next fundamental property concerns the orthogonality of the EPRs. Rewriting Eq. (21) in terms of the amplitude of the zeropoint fluctuation, we have

$\varphi_{m j}=s_{m j} \sqrt{p_{m j} \hbar \omega_{m} / 2 E_{j}}$,

where the EPR sign $s_{m j}$ of junction $j$ in mode $m$ is either +1 or -1 . The EPR sign encodes the relative direction of current flowing across the junction. Only the relative value between $s_{m j}$ and $s_{m j^{\prime}}$ for $j \neq j^{\prime}$ has physical significance (see Supplementary Fig. 8). The EPR sign $s_{m j}$ is calculated in parallel with the process of calculating $p_{m j}$, from the field solution $\vec{H}(\vec{r})$, see Supplementary Section C3. We now obtain the EPR orthogonality relationship

$\sum_{m=1}^{M} s_{m j} s_{m j^{\prime}} \sqrt{p_{m j} p_{m j^{\prime}}}=0$,

valid when the sum from 1 to $M$ covers all the relevant modes, see Supplementary Section A7.

Excitation-number-conserving interactions: Thus, as announced, knowledge of the EPRs completely specifies $\hat{H}_{\mathrm{nl}}$, through Eqs. (17), (19), and (21). The Hamiltonian can now be analytically or numerically diagonalized using various computational techniques $^{20}$. In this section, our focus will be now to explicitly handle the effect of the nonlinear interactions $\hat{H}_{\mathrm{nl}}$ on the eigenmodes. Before treating the case of a general nonlinear interaction, we focus on the leading-order effect of $\hat{H}_{\mathrm{nl}}$ in the case of the "canonical" Josephson system. In this case, the J Josephson dipoles are all Josephson tunnel junctions and the dispersive regime is satisfied for all pairs of modes $k$ and $m$; i.e., $\omega_{k}-\omega_{m} \gg$ $E_{j} c_{j p}<\hat{\varphi}_{j}^{p}>$ for $p \geq 3$ and in the absence of strong drives. The leading-order nonlinear terms are the subset of $p=4$ terms that conserve excitation number. After normal ordering, see Supplementary Section B1, one finds the effective Hamiltonian

$\hat{\bar{H}}_{4}=-\hbar \sum_{m=1}^{M} \Delta_{m} \hat{a}_{m}^{\dagger} \hat{a}_{m}+\frac{a_{m}}{2} \hat{a}_{m}^{\dagger 2} \hat{a}_{m}^{2}+\sum_{n<m} X_{m n} \hat{a}_{m}^{\dagger} \hat{a}_{n}^{\dagger} \hat{a}_{m} \hat{a}_{n}$,

which is a generalization of the one found in Eq. (8). In Eq. (25), we have introduced the Lamb shift $\Delta_{m}$ of mode $m$, the anharmonicity $a_{m}$ of the mode, and its total dispersive shift $\chi_{m n}$ (so-called crossKerr term) with a different mode, labeled $n$. Each of these parameters is directly calculated from the EPRs. As shown in Supplementary Section B, for arbitrary $m$ and $n$,

$X_{m n}=\sum_{j=1}^{\lrcorner} \frac{\hbar \omega_{m} \omega_{n}}{4 E_{j}} p_{m j} p_{n j}$

while $a_{m}=X_{m m} / 2$ and $\Delta_{m}=\sum_{n=1}^{M} X_{m n} / 2$. Equation (26) implements, mathematically, the idea that the amplitude of these nonlinear couplings is the result of a spatial-mode scalar product of the EPRs. Remarkably, from Eq. (26) it is seen that the EPRs are essentially the only free parameters subject to design, when determining the nonlinear couplings, since $\omega_{m}, \omega_{n}$ and $E_{j}$ are generally tightly constrained by experimental considerations. 
Equation (26) can be cast in matrix form by introducing the EPR matrix

$\mathbf{P}:=\left(\begin{array}{ccc}p_{11} & \cdots & p_{1 \jmath} \\ \vdots & \ddots & \vdots \\ p_{M 1} & \cdots & p_{M J}\end{array}\right)$,

which we have found useful in handling large circuits, especially for those in excess of 100 modes. We also introduce the diagonal matrices of eigenfrequencies $\Omega:=\operatorname{diag}\left(\omega_{1}, \ldots, \omega_{M}\right)$ and junction energies $\mathbf{E}_{J}:=\operatorname{diag}\left(E_{1}, \ldots, E_{J}\right)$, which lead to the matrix form of Eq. (26),

$$
\begin{aligned}
& \text { Kerr matrix: } \boldsymbol{X}=\frac{\hbar}{4} \boldsymbol{\Omega} \mathbf{P E}_{\boldsymbol{J}}^{-1} \mathbf{P} \boldsymbol{\Omega}, \\
& \text { Anharmonicity: } \boldsymbol{a}_{m}=\frac{1}{2}[\boldsymbol{X}]_{m m}, \\
& \text { Lamb shift: } \Delta_{m}=\frac{1}{2} \sum_{m^{\prime}=1}^{M}[\boldsymbol{X}]_{m m^{\prime}} .
\end{aligned}
$$

We have defined the symmetric matrix of dispersive shifts $X$, with elements $[\boldsymbol{X}]_{m m^{\prime}}=X_{m m^{\prime}}$. Further discussion of the matrix approach and applications to $p$ th-order corrections is deferred to Supplementary Section B2, and the amplitude of an arbitrary multiphoton interaction stemming from the full $\hat{H}_{\mathrm{nl}}$ is calculated in Supplementary Section B3.

EPR for dissipation in the circuit: The EPR method treats the calculation of Hamiltonian and dissipation parameters on equal footing. Unlike in the impedance method ${ }^{4}$, one can completely characterize both $\hat{H}_{\text {full }}$ and the effect of dissipative elements in the circuit from the eigenfield solutions, $\vec{E}_{m}(\vec{r})$ and $\vec{H}_{m}(\vec{r})$. The list of dissipative elements include bulk and surface dielectrics ${ }^{81-83}$ thin-film metals ${ }^{53,84}$, surface interfaces ${ }^{85-89}$, and metal seams ${ }^{90}$. The EPR of a dissipative element $/$ in mode $m$ will be denoted $p_{m /}$. It is calculated similarly to $p_{m j}$, as summarized in Supplementary Section D. The participation $p_{m l}$ and the quality factor $Q_{l}$ of the material of this element are used to estimate the total quality factor of mode $m$ in the standard way when the fields are not

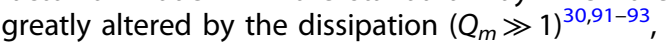

$$
Q_{m}^{-1}=\sum_{l} p_{m l} Q_{l}^{-1} \text {. }
$$

Experimental values of $Q_{l}$ are found in the literature, and some are provided in Supplementary Section D. Equation (29) and the dissipative EPR $p_{m l}$ provide a dissipation budget for the individual influence of each dissipation mechanism in the system, providing a useful tool to optimize design layout for quantum coherence ${ }^{94}$.

\section{Comparison between theory and experiment}

Applying the EPR method, we designed eight superconducting samples to test the agreement between the EPR theory and experimental results. We tested several sample configurations, comprising 15 qubits, 8 cavity modes, and 1 waveguide in 3 different circuit quantum electrodynamics (CQED) architectures. The samples were measured in a standard CQED set-up, see "Methods," at the $15 \mathrm{mK}$ stage of a dilution unit, over multiple cool downs.

Six of the samples were each composed of two qubits and one 3D cavity, one sample was composed of two qubits and a waveguide, and one sample was a flip-chip, 2.5D system ${ }^{28}$ consisting of a flip-chip qubit embedded in a two-mode whispering gallery mode resonator ${ }^{53}$ (WGMR). The specifics of each sample are discussed in the "Methods" section.

For each sample, we measured the circuit parameters of interest: dressed mode frequencies $\omega_{m}-\Delta_{m}$, anharmonicities of qubits and high- $Q$ cavities $a_{m}$, cross-Kerr frequencies $X_{m n}$, and input-output $(I-O)$ quality factors $Q_{C}$ for any readout modes. Our measurement methodology is detailed in the "Methods" section.
The measured parameters were compared to those calculated using the energy-participation method. The linearized Josephson circuit of each sample was modeled in Ansys High-Frequency Electromagnetic-Field Simulator (HFSS). Junctions were modeled as lumped inductors, whose nominal energy $E_{j}$ was inferred from roomtemperature resistance measurements ${ }^{49}$. To account for the error bars of the measurement and the drift in resistance over time, $E_{j}$ was adjusted by no more than $10 \%$ to fit the measured qubit frequency. To minimize the number of free parameters, we neglect the small junction intrinsic capacitance $C_{J}$ in our modeling. The tradeoff is a small and estimable systematic offset of the bare simulated mode anharmonicities. We estimate this correction to be on the order of $4 \%$ for a $C_{J}=4 \mathrm{fF}$. From the eigenfield solutions, we calculated the EPRs $p_{m j}$ and the sign $s_{m j}$ to construct $\hat{H}$ and extract its parameters. Detailed steps of the procedure can be found in Supplementary Section C. The results are presented in Tables 1-3.

Figure 4 summarizes the agreement of the measured and calculated sample parameters, which span five orders of magnitude in frequency. Accounting for $C_{J}$, we find that mode frequencies are calculated to $1 \%$ accuracy, large nonlinear interaction energies (namely, anharmonicity and cross-Kerr frequencies $>10 \mathrm{MHz}$ ) are calculated at the $5 \%$ level, and small nonlinear interaction energies agree at the $10 \%$ level. We highlight that we have used minimal, coarse adjustment to account for shifts in $E_{j}$ and otherwise, by neglecting $C_{j}$, the calculation is free from adjustable parameters.

The results of Fig. 4 demonstrate the accuracy and applicability of the EPR method. For each device, the EPR results are obtained from a single eigenmode simulation, using full automation of the analysis, provided by our open-source package PYEPR (see the PYEPR ${ }^{95}$ code repository at http://github.com/zlatko-minev/pyEPR). For current standard applications, we find the agreement sufficient. Further improvements in accuracy would require improved ability to estimate the Josephson dipole energy $E_{j}$ and its intrinsic capacitance $C_{j}$. At the same level of accuracy, improvements in the precision and reproducibility of the implementation and assembly of the Josephson circuit design are needed, such as in chip-clamping techniques, precision machining of the device sample holder, and $I-O$ couplers.

Conclusion. An intuitive, easy-to-use, and efficient method is needed to design and analyze Josephson microwave quantum circuits. We have described in this article such a method, based on the distribution of the electromagnetic energy in the circuit and its participation in nonlinear and dissipative elements. This so-called EPR method offers physical insight helping the design process and provides a simple link between the classical circuit and its quantum properties. By comparing our theory to eight experimental devices incorporating Josephson junctions, we have shown that our method is accurate and applicable to a large range of quantum circuit architectures. It is directly applicable to a broader class of nonlinear inductive elements, such as weak-link nanobridges 38,63 , nanowires $^{40,41,64,66,67}$, and kinetic-inductance thin films ${ }^{43,58,59}$. While best suited for weakly nonlinear systems, the EPR method is derived within circuit theory without approximations. It can be seen as arising from a change of basis adapted to nonlinear elements, as detailed in Supplementary Section A. In practice, the useful reach of the method is set by the numerical ability to include all relevant electromagnetic modes and to compute the spectrum of the extracted Hamiltonian ${ }^{20}$. We contribute an open-source package PYEPR (see the PYEPR ${ }^{95}$ code repository at http://github.com/zlatko-minev/pyEPR), which automates the EPR method, and was tested in the design of several further experiments ${ }^{28,74,96-105}$.

\section{METHODS}

\section{Methods of the experiment}

Device fabrication. Unless otherwise noted, samples were fabricated according to the following methodology. Sample patterns, both large and fine features, were defined by a $100 \mathrm{kV}$ electron-beam pattern generator 
Table 1. Two-qubit, one-cavity devices.

\begin{tabular}{|c|c|c|c|c|c|c|c|c|c|}
\hline \multirow[t]{2}{*}{ Device } & \multicolumn{3}{|c|}{ Frequency $(\mathrm{MHz})$} & \multicolumn{2}{|c|}{ Anharmonicity (MHz) } & \multicolumn{3}{|c|}{ Cross-Kerr (MHz) } & \multirow{2}{*}{$\begin{array}{l}\text { I-O coupling } \\
Q_{C}\end{array}$} \\
\hline & $\omega_{\mathrm{D}} / 2 \pi$ & $\omega_{\mathrm{B}} / 2 \pi$ & $\omega_{\mathrm{C}} / 2 \pi$ & $a_{\mathrm{D}} / 2 \pi$ & $a_{\mathrm{B}} / 2 \pi$ & $X_{\mathrm{DB}} / 2 \pi$ & $X_{\mathrm{BC}} / 2 \pi$ & $X_{D C} / 2 \pi$ & \\
\hline \multirow[t]{3}{*}{$\mathrm{R} 9 \mathrm{C} 1$} & 4951 & 5664 & 9158 & 138 & 170 & 92 & 4.7 & 0.4 & $5.20 \times 10^{3}$ \\
\hline & 4866 & 5691 & 9154 & 150 & 185 & 99 & 4.2 & 0.55 & $7.40 \times 10^{3}$ \\
\hline & $-1.7 \%$ & $0.5 \%$ & $-0.04 \%$ & $8 \%$ & $8 \%$ & $7 \%$ & $-12 \%$ & $27 \%$ & $29 \%$ \\
\hline \multirow[t]{3}{*}{$\mathrm{R} 2 \mathrm{C} 1$} & 4823 & 5567 & 8947 & 150 & 192 & 64.5 & 4.8 & 0.3 & $4.97 \times 10^{3}$ \\
\hline & 4770 & 5640 & 8950 & 161 & 211 & 67.7 & 5.88 & 0.46 & $5.44 \times 10^{3}$ \\
\hline & $-1.1 \%$ & $1.3 \%$ & $0.03 \%$ & $6.8 \%$ & $9 \%$ & $4.7 \%$ & $18 \%$ & $35 \%$ & $9 \%$ \\
\hline \multirow[t]{3}{*}{ R7C1 } & 4726 & 5475 & 8999 & 156 & 189 & 67 & 4.8 & 0.34 & $2.68 \times 10^{3}$ \\
\hline & 4770 & 5640 & 8950 & 161 & 211 & 67.7 & 5.88 & 0.46 & $3.07 \times 10^{3}$ \\
\hline & $0.9 \%$ & $2.9 \%$ & $-0.55 \%$ & $3.1 \%$ & $10 \%$ & $1 \%$ & $18 \%$ & $26 \%$ & $13 \%$ \\
\hline \multirow[t]{3}{*}{$\mathrm{R} 3 \mathrm{C} 2$} & 4845 & 5620 & 8979 & 152 & 195 & 61 & 5.1 & 0.3 & $2.11 \times 10^{3}$ \\
\hline & 4770 & 5640 & 8950 & 161 & 211 & 67.7 & 5.88 & 0.46 & $1.78 \times 10^{3}$ \\
\hline & $-1.5 \%$ & $0.4 \%$ & $-0.3 \%$ & $5.6 \%$ & $7.6 \%$ & $9.9 \%$ & $13 \%$ & $35 \%$ & $-19 \%$ \\
\hline \multirow[t]{3}{*}{$\mathrm{R} 3 \mathrm{C} 1$} & 4688 & 5300 & 9003 & 148 & 174 & 85 & 5 & 0.33 & $2.43 \times 10^{3}$ \\
\hline & 4745 & 5265 & 8922 & 159 & 198 & 73 & 5.1 & 0.37 & $5.65 \times 10^{3}$ \\
\hline & $1.2 \%$ & $-0.7 \%$ & $-0.9 \%$ & $6.9 \%$ & $12.1 \%$ & $-16 \%$ & $2 \%$ & $9 \%$ & $57 \%$ \\
\hline \multirow[t]{3}{*}{ DT3 } & 6160 & 7110 & 9170 & 130 & 150 & 278 & 3 & 2.5 & $9.17 \times 10^{3}$ \\
\hline & 6100 & 7141 & 9155 & 140 & 177 & 312 & 3.9 & 3.1 & $7.33 \times 10^{3}$ \\
\hline & $-1.0 \%$ & $0.4 \%$ & $-0.15 \%$ & $7 \%$ & $15 \%$ & $11 \%$ & $23 \%$ & $19 \%$ & $-25 \%$ \\
\hline
\end{tabular}

Summary of measured and calculated Hamiltonian and input-output $(I-O)$ coupling parameters for the six devices described in "Methods." Indices D, B, and C denote the dark, bright, and cavity modes, respectively. The input-output quality factor to the readout cavity is denoted $Q_{\mathrm{C}}$. For each device, the first (second) row quantifies the measured, $m$, (bare calculated, $c$ ) values. The third row quantifies the bare agreement, i.e., $(c-m) / c$. In the anharmonicity column, the bare agreement should be corrected by the systematic shift due to our choice to neglect the junction intrinsic capacitance in our modeling (see "Methods"). We evaluate the correction to be of order $4 \%$, estimated by taking a nominal junction $C_{J}=4 \mathrm{fF}$; hence, an overall corrected agreement of $4.3 \%$ for this column.

Table 2. Flip-chip (2.5D), one-qubit, one-storage-cavity, one-readout-cavity devices.

\begin{tabular}{|c|c|c|c|c|c|c|c|}
\hline Device & \multicolumn{3}{|c|}{ Frequency (MHz) } & $\begin{array}{l}\text { Anharmonicity }(\mathrm{MHz}) \\
a_{\mathrm{D}} / 2 \pi\end{array}$ & \multicolumn{2}{|c|}{ Cross-Kerr (MHz) } & $\begin{array}{l}\text { I-O couplins } \\
Q_{C}\end{array}$ \\
\hline \multirow[t]{3}{*}{ WG1 } & 4890 & 7070 & 7267 & 310 & 0.25 & 0.30 & $20 \times 10^{3}$ \\
\hline & 4820 & 7020 & 7340 & 325 & 0.29 & 0.33 & $16 \times 10^{3}$ \\
\hline & $-1.4 \%$ & $-0.7 \%$ & $1.0 \%$ & $4.6 \%$ & $13 \%$ & $9 \%$ & $-22 \%$ \\
\hline
\end{tabular}

Summary of measured and calculated Hamiltonian and input-output $(I-O)$ coupling parameters for the device described in "Methods." Indices $Q$, $S$, and $C$ denote the qubit, storage, and readout cavity modes, respectively. The input-output quality factor to the readout cavity is denoted $Q_{c}$. For each device, the first (second) row quantifies the measured, $m$, (bare calculated, $c$ ) values. The third row quantifies the bare agreement, i.e., $(c-m) / c$.

Table 3. Two-qubit, one-waveguide devices.

\begin{tabular}{|c|c|c|c|c|}
\hline \multicolumn{2}{|c|}{ Frequency (MHz) } & \multicolumn{2}{|c|}{ Anharmonicity $(\mathrm{MHz})$} & \multirow{2}{*}{$\begin{array}{l}\text { Cross-Kerr }(\mathrm{MHz}) \\
\chi_{\mathrm{DB}} / 2 \pi\end{array}$} \\
\hline$\omega_{\mathrm{D}} / 2 \pi$ & $\omega_{\mathrm{B}} / 2 \pi$ & $a_{\mathrm{D}} / 2 \pi$ & $a_{\mathrm{B}} / 2 \pi$ & \\
\hline 6010 & 8670 & 85 & 180 & 278 \\
\hline 5824 & 8878 & 97 & 206 & 281 \\
\hline$-3.2 \%$ & $2.3 \%$ & $12 \%$ & $13 \%$ & $1.1 \%$ \\
\hline
\end{tabular}

Summary of measured and calculated Hamiltonian parameters for the device described in "Methods." Indices D and B denote the dark and bright modes, respectively. For each device, the first (second) row summarizes the measured, $m$, (bare calculated, $c$ ) values. The third row quantifies the bare agreement, i.e., $(c-m) / c$.

(Raith EBPG 5000+) in a single step on a PMAA/MAA (Microchem A-4/ Microchem EL-13) resist bilayer coated on a $430 \mu \mathrm{m}$ thick, double-sidepolished, c-plane sapphire wafer, grown with the edge-defined film-fed growth technique. Using the bridge-free fabrication technique ${ }^{106-108}$, the
$\mathrm{Al} / \mathrm{AlO}_{x} / \mathrm{Al}$ Josephson tunnel junctions were formed by a double-angle aluminum evaporation under ultra-high vacuum in a multi-chamber Plassys UMS300 UHV. The two depositions were interrupted by a thermal oxidation step, static 100 Torr environment of $85 \%$ argon and $15 \%$ oxygen, to form the thin $\mathrm{AlO}_{x}$ barrier of the tunnel junction. Prior to the first deposition, to reduce junction aging ${ }^{108}$, the exposed wafer surfaces were exposed to 1 min oxygen-argon plasma cleaning, under a pressure of $3 \times$ $10^{-3}$ mbar. After wafer dicing (ADT ProVecturs 7100) and chip cleaning, the normal-state resistance $R_{N}$ of the Josephson junctions was measured to provide an estimate of the Josephson energy, $E_{J}$, of the device junctions. The junction energy was to first order estimated by an extrapolation of $R_{\mathrm{N}}$ from room temperature to the operating sample temperature, at approximately $15 \mathrm{mK}$, using the Ambegaokar-Baratoff relation ${ }^{109}$,

$E_{J}=\frac{1}{2} \frac{h \Delta}{(2 e)^{2}} R_{\mathrm{N}}^{-1}$

where $\Delta$ is the superconducting gap of aluminum, $e$ is the elementary charge, and $h$ is Planck's constant.

Sample holder. Sample holders were machined in aluminum alloy 6061; seams were formed using thin indium gaskets placed in machined grooves 
Theory vs. experiment

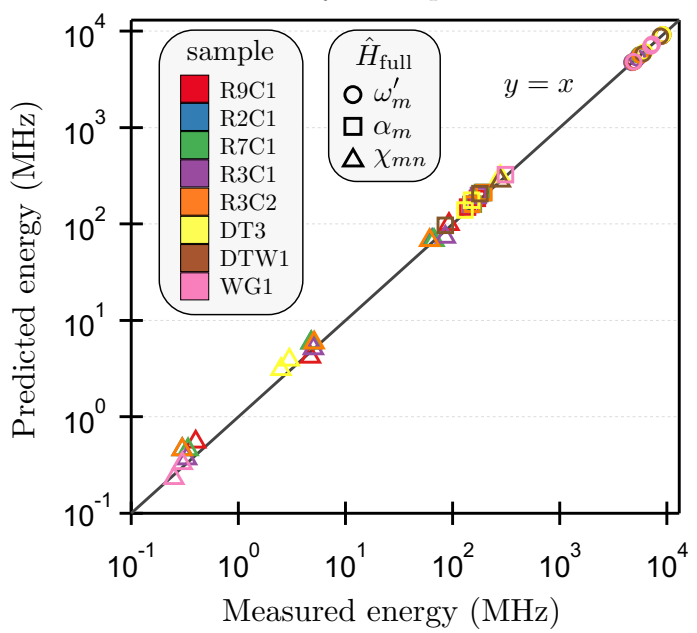

Fig. 4 Theory vs. experiment. Comparison between theory and experiment over five-orders of magnitude in energy scale of the system Hamiltonian $\hat{H}_{\text {full }}$ for eight distinct, multi-mode device samples, described in detail in the "Methods" section, including $3 \mathrm{D}$, flip-chip (2.5D), and 3D waveguide architectures incorporating readout and storage resonators and qubit modes. For each device, the dominant parameters in $\hat{H}_{\text {full }}$, dressed frequencies $\omega_{m}^{\prime}$, bare anharmonicities $a_{m}$, and cross-Kerr interactions $X_{m n}$ were measured and calculated using the EPR method with our open-source PYEPR package (see the PYEPR ${ }^{95}$ code repository at http://github.com/ zlatko-minev/pyEPR). Gray line is of slope one, representing ideal agreement between theory and experiment.

in one of the mating surfaces. Only non-magnetic components were used in proximity to the samples, molybdenum washers, aircraft-alloy 7075 screws (McMaster/Fastener Express) with $<1 \%$ iron impurities, and non-magnetic SubMiniature version A (SMA) connectors.

Cryogenic set-up. Samples were thermally anchored to the $15 \mathrm{mK}$ stage of a cryogen-free dilution refrigerator (Oxford Triton 200) and were measured using a standard CQED measurement set-up ${ }^{45,51,96}$. High-magneticpermeability, $\mu$-metal (Amumetal A4K) shields together with aluminum superconducting shields enclosed all samples. Microwave input and output lines were filtered with Eccosorb CR-110 infrared-frequency filters ${ }^{55,92}$, thermally anchored at the $15 \mathrm{mK}$ stage. Output lines were additionally filtered with cryogenic isolators (Quinstar CWJ1019-K414) and $12 \mathrm{GHz}$ K\&L multi-section lowpass filters. Output lines leading up to the high-electron-mobility transistor (HEMT) amplifier (Low Noise Factory), anchored at $4 \mathrm{~K}$, were superconducting (CoaxCo Ltd. SC-086/50-NbTi$\mathrm{NbTi}$ PTFE).

Quantum amplifier. The output signal of a sample was processed by a Josephson parametric converter (JPC) anchored at the $15 \mathrm{mK}$ stage and operated in amplification mode ${ }^{110,111}$, before routing to the HEMT. The JPC provide a typical gain of $21 \mathrm{~dB}$ with a typical noise-visibility ratio of $6 \mathrm{~dB}$. See ref. ${ }^{112}$ for a review of the parametric amplification.

Frequency and I-O coupling measurements. Spectroscopic measurements were used to determine the frequencies of the resonator modes. Anharmonicities were determined in two-tone spectroscopy 92,113 . CrossKerr energies were determined from dressed dephasing measurements ${ }^{114,115}$. In particular, the dressed-dephasing measurement sequence consisted of first preparing the qubit in the ground state, then exciting it to the equator by a $\pi / 2$ pulse. Subsequently, a weak readout tone excited the readout cavity of the qubit for a fixed duration, 10 times the readout cavity lifetime $\kappa_{\mathrm{r}}$, after which we measure the qubit $X$ and $Y$ Bloch vectors, after waiting for a time $5 / \mathrm{K}_{\mathrm{r}}$ for any photons in the cavity to leak out. By varying the amplitude and frequency of the applied weak-readout tone, we could calibrate both the strength of our readout, in steady-state photon number in the readout cavity, and the value of the cross-Kerr frequency shift between the qubit and readout resonator. The values could be obtained
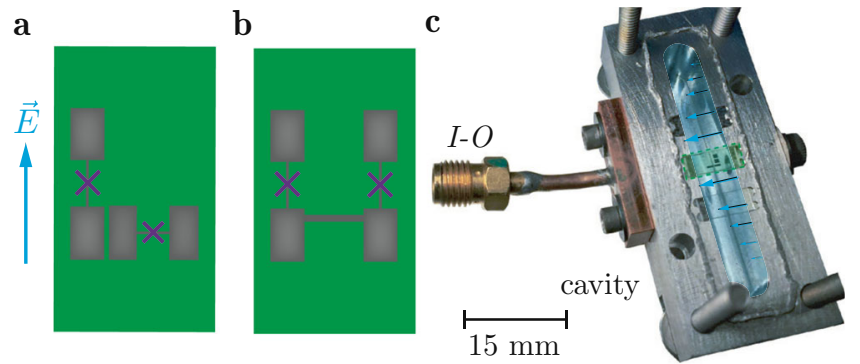

Fig. 5 Two-qubit, one-cavity devices. a, b Not-to-scale diagram illustrating chip configurations $\mathrm{A}$ and $\mathrm{B}$, respectively. Vertical blue arrow indicates cavity electric field orientation. Crosses mark the location of Josephson tunnel junctions. c Optical photograph of sample R2C1. Bottom half of aluminum sample holder is visible; top half is removed. The two-qubit chip (outlined by the dashed green box) is housed in the middle of the readout cavity (highlighted in blue). Cavity fundamental mode electric field profile $\mathrm{E}$ depicted by arrows. Input-output SMA pin coupler labeled I-O.

from fits of the $X$ and $Y$ quadratures. For each sample, the coupling quality factor of the readout-cavity mode, denoted $Q_{c}$, was extracted from the spectroscopic response of the readout cavity at low photon numbers ${ }^{92,113}$, by measuring the scattering parameters, $S_{21}$ or $S_{11}$.

To test EPR's robustness to experimental variability and its applicability over wide range of experimental conditions, the presented samples were fabricated in multiple runs and measured in different cooldowns. Some devices were subjected to as many as six thermal cycles.

The Hamiltonian parameters and coupling energies for each sample were also calculated, following the EPR method presented in section on the general approach. In particular, we modeled the sample geometry and materials in a FE electromagnetic simulation, as explicated in Supplementary Section C. Our aim in writing this supplementary section has been to provide an easy access point to the practical use of the EPR method, which we hope will benefit the reader, and allow them to adopt it easily. Our choice of simulation software was the Ansys HFSS, although we emphasize that the EPR ideas translate to any standard EM eigenmode simulation package. Further, we modeled the loss due to the $I-O$ couplers in the simulation as $50 \Omega$ resistive sheets, see Supplementary Section D4. The eigenmode analysis provided the calculated $I-O$ quality factors and Purcell limits. All electromagnetic and quantum analyses, including the extraction of participations from the eigenfields and the numerical diagonalization of the Hamiltonian to extract its quantum spectrum, were performed in a fully automated manner using the freely available PYEPR package (see the PYEPR $^{95}$ code repository at http://github.com/zlatko-minev/pyEPR).

The mode quality due to the $I-O$ coupling, $Q_{C}$, was set by the length of the I-O SMA-coupler pin. Its length inside the sample-holder box was measured at room temperature using calipers. This nominal length was used then used in the HFSS model to create a 3D model of the pin inside the sample holder. The quality factor $Q_{C}$ was then obtained from the eigenmode eigenvalue. We remark that the measurement of the pin length is accurate to no more than 20\%; further, it can be affected by various idiosyncrasies, such as bending of the thin SMA center pin. Nonetheless, the predictions of the quality factors for low- $Q$ modes were observed to be very reasonable estimates, and similarly, the predicted Purcell limits for qubit and high- $Q$ cavity modes were consistent with estimates from measurements.

\section{Devices: two-qubit, one-cavity devices}

Device description. We measured six samples that were each comprised of two qubits and one cavity. The cavity was a standard, machined aluminum cavity $^{54}$. It housed either one or two sapphire chips, which were either patterned with transmon qubits or simply blank. Each transmon consisted of two thin-film aluminum pads connected by a Josephson junction. We tested two configurations of chips and patterns. Configuration A consisted of one chip with two orthogonal qubits, as depicted in Fig. 5a. Similarly, configuration B consisted of one chip with two parallel qubits, depicted in Fig. $5 \mathrm{~b}$. The two qubits were aligned parallel to each other; however, unlike configuration $B$, there was no galvanic connection between them. The results of the measurements are presented in Table 1. 
Samples $\mathrm{R} 1 \mathrm{C} 9, \mathrm{R} 2 \mathrm{C} 1, \mathrm{R} 7 \mathrm{C} 1, \mathrm{R} 2 \mathrm{C} 1$, and $\mathrm{R} 3 \mathrm{C} 2$ were fabricated in configuration $\mathrm{A}$; sample DT3 was fabricated in configuration $\mathrm{B}$. Three of the sample ( $\mathrm{R} 2 \mathrm{C} 1, \mathrm{R} 7 \mathrm{C} 1, \mathrm{R} 3 \mathrm{C} 1)$ were fabricated simultaneously on the same sapphire wafer, all with nominally identical dimensions. Additionally, $\mathrm{R} 2 \mathrm{C} 1$ and $\mathrm{R} 7 \mathrm{C} 1$ were designed to have nominally the same Josephson junctions energy, $E_{j}$. The rest of the samples (R1C9, DT3, and R3C2) were fabricated at different times and on different wafers. The dimensions of their transmons and the inductance of the junctions were designed to be different. Only sample $\mathrm{R} 3 \mathrm{C} 2$ was designed to be very similar to the nominally identical sample $\mathrm{R} 2 \mathrm{C} 1$ and $\mathrm{R} 7 \mathrm{C} 1$ but with adjusted $E_{j}$. For samples $\mathrm{R} 2 \mathrm{C} 1, \mathrm{R} 7 \mathrm{C} 1, \mathrm{R} 3 \mathrm{C} 1$, and $\mathrm{R} 3 \mathrm{C} 2$ a second, un-processed, unpatterned, blank sapphire chip was placed in parallel with the qubit carrying chip [see Fig. 5c] to purposefully lower the readout cavity frequency, thus bringing it within the JPC amplification band.

Configurations $A$ and $B$ were designed to test the ability of the EPR method to calculate the mixing between strongly coupled modes. The strong coupling was achieved in two distinct ways. First, configuration A used the spatial proximity of the two qubits to yield a strong capacitive coupling between them, which resulted in large qubit-qubit mixing. Second, instead of spatial proximity, configuration B used a galvanic connection between the qubits to yield strong hybridization. Our twoqubit designs share some similar-in-spirit characteristics with the promising recent developments reported in refs. ${ }^{116-121}$, but our implementation is distinct and is designed to provide several unique advantages.

Mode structure and interesting physical insights. Configuration $A$ is characterized by strong capacitive coupling between the two transmons, which have different pad sizes, see Fig. $5 c$, and hence different normalmode frequencies. Due to the strong hybridization, each qubit normal mode consists of some excitation in the vertical and some in the horizontal transmon. With some foresight, we will label the vertical mode bright (B) and the horizontal dark (D). The bright-mode resonance is higher in frequency and thus is closer to the resonance of the readout cavity mode (C). This smaller detuning made it a natural choice for designing stronger coupling between it, (B), and the readout mode (C). This was implemented by orienting the transmon design that participates in mode (B) vertical.

To understand this design choice, let us first consider the popular analogy 46,122 between CQED and cavity QED, often used to discuss mode couplings. In this atomic analogy, the transmon qubit is analogous to a real atom inside the cavity. Thus, it can described by an electric-dipole moment $\vec{d}_{B}$. Meanwhile, its coupling, cross-Kerr, etc. to the cavity mode are derived from the electric-dipole coupling interaction. In particular, the coupling amplitude is proportional to $\vec{d}_{B} \cdot \vec{E}$, where $\vec{E}$ is the cavity electric field at the transmon junction. From this analogy, one can infer that the coupling is maximized when the two are parallel, $\vec{d}_{B} \| \vec{E}$, and one could hope to measure a strong cross-Kerr between the bright qubit and the cavity. This successful conclusion is true, but a coincidence. We will shortly discuss how this popular analogy fails spectacularly for the dark mode in configuration B. Instead, we will argue that a correct way to understand the nonlinear coupling between the two modes is through the participation ratio, which will provide the correct coupling for both configurations $A$ and $B$.

Before proceeding to configuration $B$, we note one further useful features that configuration A exhibits. In particular, while the bright qubit mode can be Purcell limited ${ }^{116,123}$, the dark mode is simultaneously Purcell protected. Thus, one can potentially achieve a high ratio in the $I-O$ bath coupling of the two qubits.

Configuration $B$ has two qubit modes, which we will also label dark (D) and bright (B). Since both transmons are designed with the exact same transmon pad geometry and junction energy $E_{J}$, see Fig. 5 , we can expect that no single junction is preferred, due to the symmetry of the sample. This is in sharp contrast to the asymmetric energy distribution in configuration A. Returning to configuration B, we can estimate that, in each qubit mode, both junctions participate equally and with near maximal allowed participation,

$p_{\mathrm{D} 1}=p_{\mathrm{D} 2}=p_{\mathrm{B} 1}=p_{\mathrm{B} 2} \approx \frac{1}{2}$

If the two transmons were well-separated spatially and not connected, they would be uncoupled. However, the galvanic connection between the two lower pads, see Fig. 5 a, results in a very strong hybridization and splitting between the nominally identical transmons. The result of the strong hybridization is a symmetric and antisymmetric combination of the two bare transmons. In other words, the hybridization results in a common mode, namely, (B), where both junctions oscillate in phase, and a differential mode, namely, (D), where both junctions oscillate out of phase. These phase relationships are captured by the signs:

$s_{\mathrm{D} 1}=1, \quad s_{\mathrm{D} 2}=-1$,

$s_{\mathrm{B} 1}=1, \quad s_{\mathrm{B} 2}=+1$

In an attempt to understand how these hybridized qubit modes will couple to the cavity mode $(\mathrm{C})$, let us first consider the atomic analogy again. When the two junctions oscillate in phase, in the (B) mode, the net dipole moment of the bright mode, $\vec{d}_{B}$, must be large, since it is the sum of the two junction dipole contributions. Second, $\vec{d}_{\mathrm{B}}$ must be oriented in the vertical direction, parallel to the cavity electric field $\vec{E}$. Hence, we would conclude that the bright mode coupling is large, $\vec{d}_{\mathrm{B}} \cdot \vec{E} \gg 0$, and there should be a strong cross-Kerr interaction between the cavity and bright qubit. Continuing the analogy in the case of the dark mode, we would deduce that the net dipole moment of the dark mode is zero, since the two junctions oscillate out of phase and cancel each other's contribution, $\vec{d}_{\mathrm{D}}=0$. Thus, we should not expect any coupling between the dark qubit and the cavity mode, $\vec{d}_{\mathrm{D}} \cdot \vec{E}=0$. To the contrary of this conclusion, as can be seen in the measured results in Table 1, the nonlinear coupling of the dark and bright qubit to the cavity is nearly equal. The atomic analogy and the dipole argument have failed completely. We can understand the origin of this failure and how to arrive at the correct conclusion by using the EPR. As embodied in Eq. (26), in the dispersive regime, the nonlinear coupling between two modes, in this case a qubit and cavity, is given by the overlap of the EPR distribution. In particular, the cross-Kerr amplitude between the dark qubit and the readout cavity mode is given by

$X_{\mathrm{DC}}=\frac{\hbar \omega_{D} \omega_{\mathrm{C}}}{4 E_{J}}\left(p_{\mathrm{D} 1} p_{\mathrm{C} 1}+p_{\mathrm{D} 2} p_{\mathrm{C} 2}\right)$

where both junctions have the same junction energy $E_{J}$, and $\omega_{\mathrm{B}}$ (resp: $\left.\omega_{C}\right)$ denotes the dark qubit (resp: cavity) mode frequency. The signs, used in the atomic dipole logic, do not factor into the coupling, because the Josephson mechanics is fundamentally different. To obtain $X_{B C}$, one can replace the label " $D$ " with " $B$ " in Eq. 34. Then, it is easy to use Eq. (31) to show that the ratio of two Kerr couplings is not zero, but rather of order unity,

$X_{\mathrm{BC}} / X_{\mathrm{DC}}=\omega_{\mathrm{B}} / \omega_{\mathrm{D}}$.

Failure of the conventional dipole approach. We showed that, although the heuristic atomic analogy seems seductively accurate, it fails completely in some cases to predict the nonlinear couplings. Instead, one can use the intuition and calculation method provided by the EPRs.

As an added note, we observe that Eqs. (32) and (33) embodies the orthogonality of the participations, see Eq. (24). We also remark that, although the atomic analogy fails in the case of the nonlinear couplings, it can yield some guidance when considering the linear mixing of the modes, useful for discussing the Purcell effect. To illustrate, let us briefly extend the atomic analogy. The dipole-like coupling between the bright mode and the cavity suggests that the bright mode will inherit some coupling to the environment, mediated by the cavity. Thus, since $\vec{d}_{B} \cdot \vec{E} \gg 0$, we can expect the bright qubit to potentially be Purcell limited. In contrast, since $\vec{d}_{\mathrm{D}} \cdot \vec{E}=0$, we could expect the dark qubit to be Purcell protected. Both of these qualitative Purcell predictions are valid, but to quantify them, we will use the EPR method and FE eigenmode simulation of the sample, as will be discussed shortly.

Experimental results. Table 1 summarizes the results of the agreement between the measured and calculated Hamiltonian parameters for all twoqubit, one-cavity samples. The three modes in each sample are labeled dark (D), bright (B), and cavity (C); the reason for this convention is described above. In all samples, the qubits were designed to be in the dispersive regime with respect to the cavity, which was detuned by 2-4 GHz. However, in a large number of the samples, the two qubits were strongly hybridized, often necessitating higher-order nonlinear corrections to be included in the calculation. This strong hybridization was used as a test of the theory in this more challenging and fickle regime.

In total, for each sample we measured and calculated eight frequency parameters and one dimensionless, coupling quality factor, $Q_{C}$, of the readout cavity mode. In particular, in the low-excitation limit, the nonlinear interactions among the modes were characterized by the effective 


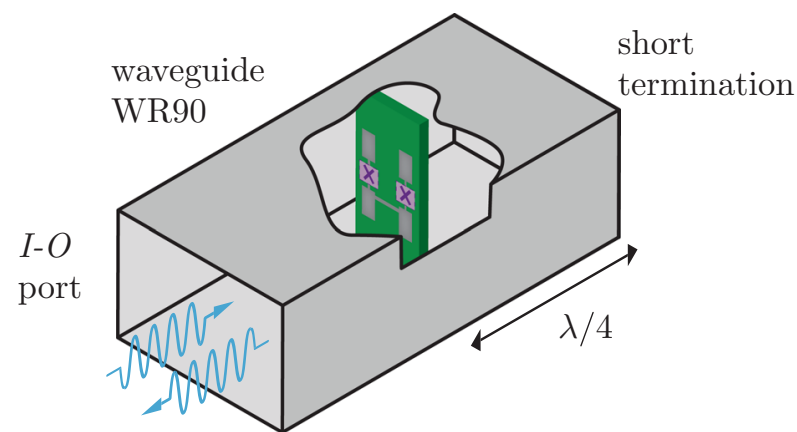

Fig. 6 Schematic representation (not to scale) of superconducting waveguide device DTW1. Two-qubit chip is placed $\lambda / 4$ from a short termination in the waveguide. Guided input-output waves are launched and monitored through the $I-O$ port connecting to a standard SMA adapter (not shown).

dispersive Hamiltonian

$$
\begin{aligned}
\hat{\bar{H}} / \hbar= & \omega_{\mathrm{D}} \hat{n}_{\mathrm{D}}+\omega_{\mathrm{B}} \hat{n}_{\mathrm{B}}+\omega_{\mathrm{C}} \hat{n}_{\mathrm{C}} \\
& -\frac{1}{2} a_{\mathrm{D}} \hat{n}_{\mathrm{D}}\left(\hat{n}_{\mathrm{D}}-\hat{1}\right)-\frac{1}{2} a_{\mathrm{B}} \hat{n}_{\mathrm{B}}\left(\hat{n}_{\mathrm{B}}-\hat{1}\right) \\
& -X_{\mathrm{DB}} \hat{n}_{\mathrm{D}} \hat{n}_{\mathrm{B}}-X_{\mathrm{DC}} \hat{n}_{\mathrm{D}} \hat{n}_{\mathrm{C}}-X_{\mathrm{B} C} \hat{n}_{\mathrm{B}} \hat{n}_{\mathrm{C}},
\end{aligned}
$$

where $\hat{n}_{\mathrm{D}}, \hat{n}_{\mathrm{B}}$, and $\hat{n}_{\mathrm{C}}$ denote the dark, bright, and cavity photon-number operator, respectively. The coupling of the resonator mode to the bath is given by the Lindblad superoperator term $\kappa_{C} \mathcal{D}\left[\hat{a}_{C}\right] \rho$, where $\kappa_{C}=\omega_{C} / Q_{C}$, and $\rho$ is the density operator.

We remark that all samples in configuration A demonstrated a large asymmetry in the Kerr coupling between the bright-to-cavity and dark-tocavity coupling, $X_{\mathrm{BC}} \gg X_{\mathrm{DC}}$. In contrast, samples in configuration $\mathrm{B}$ demonstrated near equal coupling, $X_{\mathrm{BC}} \approx X_{\mathrm{DC}}$. In both configurations $\mathrm{A}$ and $B$, the dark mode was Purcell protected, we calculated a Purcell coupling factor of $Q_{\text {Purcell }}^{D} \gg 10^{7}$, using the eigenmode method described in Supplementary Section D4. On the other hand, the bright mode was somewhat Purcell limited, $Q_{\text {Purcell }}^{B} \approx 10^{6}$. From the relative Rabi amplitudes of the dark and bright qubits, we could verify the order of magnitude scaling calculated for the Purcell effect.

\section{Devices: two-qubit, single-waveguide devices}

We measured a two-qubit sample inside of a waveguide. Figure 6 presents the set-up and depicts the sample, which was of the configuration B type presented in Fig. 5. The sample chip was positioned inside an aluminum WR90 waveguide. The waveguide was terminated in a short at one side and attached to an impedance-matched SMA coupler port on the input-launcher side, which was used to drive and measure the waveguide. The chip was centered inside the cross-section of the waveguide and placed $\lambda / 4$ away from the termination wall, at the measurement frequency. The rest of the experimental set-up was identical to that described in section "Methods of the experiment." The two qubit modes were labeled dark and bright, similarly to the samples discussed in the section "Two-qubit, one-cavity devices."

Table 3 presents the agreement between the measured and calculated key Hamiltonian parameters of the sample. These consist of the two-mode frequencies, two-qubit anharmonicities, and the strong cross-Kerr interaction between the two qubits.

\section{Devices: flip-chip (2.5D), one-qubit, one-storage-cavity, one- readout-cavity devices}

We also designed a multilayer planar ${ }^{28}$ (2.5D) CQED sample, depicted in Fig. 7, with the EPR method. It consisted of high-Q storage mode (S), one low- $Q$ readout cavity $(C)$, and one control transmon qubit $(\mathrm{Q})$. The two cavity modes were formed in the footprint of a single WGMR ${ }^{53}$. The three modes were in the dispersive regime, and the storage mode was used to encode and decode quantum information, as well as to observe parity revivals. Details of the sample design have been reported in ref. ${ }^{28}$. The agreement between the measured parameters of the sample and those obtained by the EPR calculation methods are presented in Table 2 .

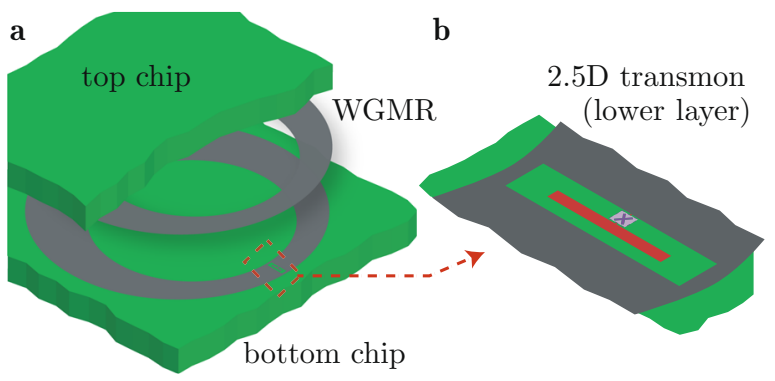

Fig. 7 Illustration of flip-chip (2.5D) device WG1. a Depiction (not to scale) of chip stack consisting of two chips separated by a $100 \mu \mathrm{m}$ vacuum gap. The inner face of each chip supports part of the pattern of a multi-layer whispering gallery-mode resonator (WGMR) resonator $^{53}$. The lower layer contains a 2.5D, aperture transmon qubit ${ }^{28}$ embedded in the WGMR. $\mathbf{b}$ Zoomed-in view of the lower layer of the aperture transmon. The cross marks the location of the Josephson tunnel junction device, which connects the lower center trace to an island embedded in the lower aperture. The capacitance to the top layer significantly participates in determining qubit parameters.

\section{DATA AVAILABILITY}

Data are available from the authors on reasonable request.

\section{CODE AVAILABILITY}

The source code for the EPR method is open-sourced and can be found at http:// github.com/zlatko-minev/pyEPR.

Received: 22 October 2020; Accepted: 17 June 2021; Published online: 25 August 2021

\section{REFERENCES}

1. Devoret, M. H. \& Schoelkopf, R. J. Superconducting circuits for quantum information: an outlook. Science 339, 1169-1174 (2013).

2. Arute, F. et al. Quantum supremacy using a programmable superconducting processor. Nature 574, 505-510 (2019).

3. Blais, A., Grimsmo, A. L., Girvin, S. M. \& Wallraff, A. Circuit quantum electrodynamics. https://journals.aps.org/rmp/abstract/10.1103/RevModPhys.93.025005 (2020).

4. Nigg, S. E. et al. Black-box superconducting circuit quantization. Phys. Rev. Lett 108, 240502 (2012)

5. Bourassa, J., Beaudoin, F., Gambetta, J. M. \& Blais, A. Josephson-junctionembedded transmission-line resonators: from Kerr medium to in-line transmon. Phys. Rev. A 86, 013814 (2012).

6. Solgun, F., Abraham, D. W. \& DiVincenzo, D. P. Blackbox quantization of superconducting circuits using exact impedance synthesis. Phys. Rev. B 90, 134504 (2014).

7. Solgun, F. \& DiVincenzo, D. P. Multiport impedance quantization. Ann. Phys. 361, 605-669 (2015).

8. Smith, W. C. et al. Quantization of inductively shunted superconducting circuits. Phys. Rev. B 94, 144507 (2016).

9. Gely, M. F. et al. Convergence of the multimode quantum Rabi model of circuit quantum electrodynamics. Phys. Rev. B 95, 245115 (2017).

10. Malekakhlagh, M., Petrescu, A. \& Türeci, H. E. Cutoff-free circuit quantum electrodynamics. Phys. Rev. Lett. 119, 073601 (2017).

11. Pechal, M. \& Safavi-Naeini, A. H. Millimeter-wave interconnects for microwavefrequency quantum machines. Phys. Rev. A 96, 042305 (2017).

12. Parra-Rodriguez, A., Egusquiza, I. L., DiVincenzo, D. P. \& Solano, E. Canonical circuit quantization with linear nonreciprocal devices. Phys. Rev. B 99, 014514 (2019).

13. Parra-Rodriguez, A., Rico, E., Solano, E. \& Egusquiza, I. L. Quantum networks in divergence-free circuit QED. Quantum Sci. Technol. 3, 024012 (2018).

14. Ansari, M. H. Superconducting qubits beyond the dispersive regime. Phys. Rev. $B$ 100, 024509 (2019).

15. Krupko, Y. et al. Kerr nonlinearity in a superconducting Josephson metamaterial. Phys. Rev. B 98, 094516 (2018).

16. Malekakhlagh, M., Petrescu, A. \& Türeci, H. E. Lifetime renormalization of weakly anharmonic superconducting qubits. I. Role of number nonconserving terms. Phys. Rev. B 101, 134509 (2020).

17. Solgun, F., DiVincenzo, D. P. \& Gambetta, J. M. Simple impedance response formulas for the dispersive interaction rates in the effective Hamiltonians of low 
anharmonicity superconducting qubits. IEEE Trans. Microwave Theory Tech. 67, 928-948 (2019).

18. Petrescu, A., Malekakhlagh, M. \& Türeci, H. E. Lifetime renormalization of driven weakly anharmonic superconducting qubits: II. The readout problem. Phys. Rev. B 101, 134510 (2019).

19. You, X., Sauls, J. A. \& Koch, J. Circuit quantization in the presence of timedependent external flux. Phys. Rev. B 99, 174512 (2019).

20. Di Paolo, A., Baker, T. E., Foley, A., Sénéchal, D. \& Blais, A. Efficient modeling of superconducting quantum circuits with tensor networks. Preprint at https:// www.nature.com/articles/s41534-020-00352-4 (2019).

21. Menke, T. et al. Automated design of superconducting circuits and its application to 4-local couplers. npj Quantum Inf. 7, 49 (2021).

22. Gely, M. F. \& Steele, G. A. QuCAT: quantum circuit analyzer tool in Python. N. J. Phys. 22, 013025 (2020).

23. Kyaw, T. H. et al. Quantum computer-aided design: digital quantum simulation of quantum processors. Preprint at http://arxiv.org/abs/2006.03070 (2020).

24. Yan, F. et al. Engineering framework for optimizing superconducting qubit designs (2020). Preprint at http://arxiv.org/abs/2006.04130. 2006.04130.

25. Minev, Z. K., McConkey, T. G., Takita, M., Corcoles, A. D. \& Gambetta, J. M. Circuit quantum electrodynamics (CQED) with modular quasi-lumped models (2021). Preprint at http://arxiv.org/abs/2103.10344.

26. Minev, Z. K. et al. Qiskit metal: an open-source framework for quantum device design \& analysis (Q-EDA). zenodo. https://zenodo.org/record/4618154 (2021).

27. Barends, R. et al. Coherent josephson qubit suitable for scalable quantum integrated circuits. Phys. Rev. Lett. 111, 080502 (2013).

28. Minev, Z. et al. Planar multilayer circuit quantum electrodynamics. Phys. Rev. Appl. 5, 044021 (2016).

29. Brecht, T. et al. Multilayer microwave integrated quantum circuits for scalable quantum computing. npj Quantum Inf. 2, 16002 (2016).

30. Reagor, M. et al. Quantum memory with millisecond coherence in circuit QED. Phys. Rev. B 94, 014506 (2016).

31. Gambetta, J. M., Chow, J. M. \& Steffen, M. Building logical qubits in a superconducting quantum computing system. npj Quantum Inf. 3, 2 (2017).

32. Rosenberg, D. et al. 3D integrated superconducting qubits. npj Quantum Inf. 3, 42 (2017).

33. Versluis, R. et al. Scalable quantum circuit and control for a superconducting surface code. Phys. Rev. Appl. 8, 034021 (2017).

34. Naik, R. K. et al. Random access quantum information processors using multimode circuit quantum electrodynamics. Nat. Commun. 8, 1904 (2017).

35. Krantz, P. et al. A quantum engineer's guide to superconducting qubits. Appl. Phys. Rev. 6, 021318 (2019).

36. Kjaergaard, M. et al. Superconducting qubits: current state of play. Annu. Rev. Condens. Matter Phys. 11, 369-395 (2020).

37. Josephson, B. Possible new effects in superconductive tunnelling. Phys. Lett. 1, 251-253 (1962).

38. Vijay, R., Levenson-Falk, E. M., Slichter, D. H. \& Siddiqi, I. Approaching ideal weak link behavior with three dimensional aluminum nanobridges. Appl. Phys. Lett. 96, 223112 (2010).

39. Kerman, A. J. Metastable superconducting qubit. Phys. Rev. Lett. 104, 027002 (2010).

40. Larsen, T. W. et al. Semiconductor-nanowire-based superconducting qubit. Phys. Rev. Lett. 115, 127001 (2015)

41. De Lange, G. et al. Realization of microwave quantum circuits using hybrid superconducting-semiconducting nanowire Josephson elements. Phys. Rev. Lett. 115, 127002 (2015).

42. Janvier, C. et al. Coherent manipulation of Andreev states in superconducting atomic contacts. Science 349, 1199-1202 (2015).

43. Maleeva, N. et al. Circuit quantum electrodynamics of granular aluminum resonators. Nat. Commun. 9, 3889 (2018).

44. Wang, J. I.-J. et al. Coherent control of a hybrid superconducting circuit made with graphene-based van der Waals heterostructures. Nat. Nanotechnol. 14, 120-125 (2019).

45. Minev, Z. K. Catching and Reversing a Quantum Jump Mid-Flight. PhD thesis, Yale Univ. (2019).

46. Koch, J. et al. Charge-insensitive qubit design derived from the Cooper pair box. Phys. Rev. A 76, 42319 (2007).

47. Yurke, B. \& Denker, J. S. Quantum network theory. Phys. Rev. A 29, 1419-1437 (1984).

48. Devoret, M. H. Quantum fluctuations in electrical circuits. In A UJFG/a NATO Advanced Study Institute Conference (eds Reynaud, S., Giacobino, E. \& Zinn-Justin, J.) 351 (Elsevier, 1997).

49. Gloos, K., Poikolainen, R. S. \& Pekola, J. P. Wide-range thermometer based on the temperature-dependent conductance of planar tunnel junctions. Appl. Phys. Lett. 77, 2915 (2000)
50. Blais, A., Huang, R.-S., Wallraff, A., Girvin, S. M. \& Schoelkopf, R. J. Cavity quantum electrodynamics for superconducting electrical circuits: an architecture for quantum computation. Phys. Rev. A 69, 062320 (2004).

51. Wallraff, A. et al. Strong coupling of a single photon to a superconducting qubit using circuit quantum electrodynamics. Nature 431, 162-167 (2004).

52. Yan, F. et al. The flux qubit revisited to enhance coherence and reproducibility. Nat. Commun. 7, 12964 (2016).

53. Minev, Z., Pop, I. M. \& Devoret, M. H. Planar superconducting whispering gallery mode resonators. Appl. Phys. Lett. 103, 142604 (2013).

54. Paik, H. et al. Observation of high coherence in Josephson junction qubits measured in a three-dimensional circuit QED architecture. Phys. Rev. Lett. 107, 240501 (2011).

55. Rigetti, C. et al. Superconducting qubit in a waveguide cavity with a coherence time approaching 0.1 ms. Phys. Rev. B 86, 100506 (2012)

56. Axline, C. et al. An architecture for integrating planar and 3D CQED devices. Appl. Phys. Lett. 109, 42601 (2016).

57. Yurke, B. \& Buks, E. Performance of cavity-parametric amplifiers, employing kerr nonlinearites, in the presence of two-photon loss. J. Lightwave Technol. 24, 5054-5066 (2006).

58. Ho Eom, B., Day, P. K., LeDuc, H. G. \& Zmuidzinas, J. A wideband, low-noise superconducting amplifier with high dynamic range. Nat. Phys. 8, 623-627 (2012).

59. Vissers, M. R. et al. Frequency-tunable superconducting resonators via nonlinear kinetic inductance. Appl. Phys. Lett. 107, 062601 (2015).

60. Mortensen, H. L., Mølmer, K. \& Andersen, C. K. Normal modes of a superconducting transmission-line resonator with embedded lumped element circuit components. Phys. Rev. A 94, 053817 (2016).

61. Koops, M. C., van Duyneveldt, G. V. \& de Bruyn Ouboter, R. Direct observation of the current-phase relation of an adjustable superconducting point contact. Phys. Rev. Lett. 77, 2542-2545 (1996).

62. Bretheau, L., Girit, Ç. Ö., Pothier, H., Esteve, D. \& Urbina, C. Exciting Andreev pairs in a superconducting atomic contact. Nature 499, 312-315 (2013).

63. Peltonen, J. T. et al. Coherent dynamics and decoherence in a superconducting weak link. Phys. Rev. B 94, 180508 (2016).

64. Mooij, J. E. \& Nazarov, Y. V. Superconducting nanowires as quantum phase-slip junctions. Nat. Phys. 2, 169-172 (2006).

65. Ku, J., Manucharyan, V. \& Bezryadin, A. Superconducting nanowires as nonlinear inductive elements for qubits. Phys. Rev. B 82, 134518 (2010).

66. Abay, S. et al. Charge transport in InAs nanowire Josephson junctions. Phys. Rev. B 89, 214508 (2014).

67. Casparis, L. et al. Gatemon benchmarking and two-qubit operations. Phys. Rev. Lett. 116, 150505 (2016).

68. Shim, Y.-P. \& Tahan, C. Bottom-up superconducting and Josephson junction devices inside a group-IV semiconductor. Nat. Commun. 5, 4225 (2014).

69. Zimmerman, J. E. \& Silver, A. H. Macroscopic quantum interference effects through superconducting point contacts. Phys. Rev. 141, 367-375 (1966).

70. Clarke, J. \& Braginski, A. I. (eds) The SQUID Handbook (Wiley-VCH Verlag, 2004).

71. Frattini, N. E. et al. 3-Wave mixing Josephson dipole element. Appl. Phys. Lett. 110, 222603 (2017)

72. Manucharyan, V. E. et al. Evidence for coherent quantum phase slips across a Josephson junction array. Phys. Rev. B 85, 024521 (2012).

73. Pop, I. M. et al. Coherent suppression of electromagnetic dissipation due to superconducting quasiparticles. Nature 508, 369-372 (2014).

74. Muppalla, P. R. et al. Bistability in a mesoscopic Josephson junction array resonator. Phys. Rev. B 97, 024518 (2018).

75. Corlevi, S., Guichard, W., Hekking, F. W. J. \& Haviland, D. B. Phase-charge duality of a Josephson junction in a fluctuating electromagnetic environment. Phys. Rev. Lett. 97, 096802 (2006).

76. Hutter, C., Tholén, E. A., Stannigel, K., Lidmar, J. \& Haviland, D. B. Josephson junction transmission lines as tunable artificial crystals. Phys. Rev. B 83, 014511 (2011).

77. Masluk, N. A., Pop, I. M., Kamal, A., Minev, Z. K. \& Devoret, M. H. Microwave characterization of Josephson junction arrays: implementing a low loss superinductance. Phys. Rev. Lett. 109, 137002 (2012).

78. Bell, M. T., Sadovskyy, I. A., loffe, L. B., Kitaev, A. Y. \& Gershenson, M. E. Quantum superinductor with tunable nonlinearity. Phys. Rev. Lett. 109, 137003 (2012).

79. Weißl, T. et al. Kerr coefficients of plasma resonances in Josephson junction chains. Phys. Rev. B 92, 104508 (2015).

80. Macklin, C. et al. A near-quantum-limited Josephson traveling-wave parametric amplifier. Science 350, 307-310 (2015).

81. Martinis, J. M. et al. Decoherence in Josephson qubits from dielectric loss. Phys. Rev. Lett. 95, 210503 (2005).

82. Patel, U. et al. Coherent Josephson phase qubit with a single crystal silicon capacitor. Appl. Phys. Lett. 102, 012602 (2013).

83. Dial, O. et al. Bulk and surface loss in superconducting transmon qubits. Supercond. Sci. Technol. 29, 044001 (2016). 
84. Vissers, M. R., Weides, M. P., Kline, J. S., Sandberg, M. \& Pappas, D. P. Identifying capacitive and inductive loss in lumped element superconducting hybrid titanium nitride/aluminum resonators. Appl. Phys. Lett. 101, 022601 (2012).

85. Wenner, J. et al. Surface loss simulations of superconducting coplanar waveguide resonators. Appl. Phys. Lett. 99, 113513 (2011).

86. Geerlings, K. et al. Improving the quality factor of microwave compact resonators by optimizing their geometrical parameters. Appl. Phys. Lett. 100, 192601 (2012).

87. Sandberg, M. et al. Long-lived, radiation-suppressed superconducting quantum bit in a planar geometry. Appl. Phys. Lett. 102, 072601 (2012).

88. Wang, C. et al. Surface participation and dielectric loss in superconducting qubits. Appl. Phys. Lett. 107, 162601 (2015).

89. Bruno, A. et al. Reducing intrinsic loss in superconducting resonators by surface treatment and deep etching of silicon substrates. Appl. Phys. Lett. 106, 182601 (2015).

90. Brecht, T. et al. Demonstration of superconducting micromachined cavities. Appl. Phys. Lett. 107, 192603 (2015).

91. Gao, J. The Physics of Superconducting Microwave Resonators. PhD thesis, California Institute of Technology (2008).

92. Geerlings, K. L. Improving Coherence of Superconducting Qubits and Resonators. PhD thesis, Yale Univ. (2013).

93. Brecht, T. et al. Micromachined integrated quantum circuit containing a superconducting qubit. Phys. Rev. Appl. 7, 044018 (2017).

94. Martinis, J. M. \& Megrant, A. UCSB final report for the CSQ program: review of decoherence and materials physics for superconducting qubits. Preprint at http://arxiv.org/abs/1410.5793 (2014)

95. Minev, Z. K. et al. pyEPR: the energy-participation-ratio (EPR) open-source framework for quantum device design. zenodo https://doi.org/10.5281/zenodo.4744447 (2021).

96. Leghtas, Z. et al. Confining the state of light to a quantum manifold by engineered two-photon loss. Science 347, 853-857 (2015).

97. Mundhada, S. O. et al. Generating higher-order quantum dissipation from lowerorder parametric processes. Quantum Sci. Technol. 2, 024005 (2017).

98. Touzard, S. et al. Coherent oscillations inside a quantum manifold stabilized by dissipation. Phys. Rev. X 8, 021005 (2018).

99. Campagne-lbarcq, P. et al. Deterministic remote entanglement of superconducting circuits through microwave two-photon transitions. Phys. Rev. Lett 120, 200501 (2018).

100. Wang, Z. et al. Cavity attenuators for superconducting qubits. Phys. Rev. Appl. 11, 014031 (2019)

101. Grimm, A. et al. The Kerr-Cat qubit: stabilization, readout, and gates. https:// www.nature.com/articles/s41586-020-2587-z (2019).

102. Minev, Z. K. et al. To catch and reverse a quantum jump mid-flight. Nature 570, 200-204 (2019).

103. Campagne-lbarcq, P. et al. A stabilized logical quantum bit encoded in grid states of a superconducting cavity. https://www.nature.com/articles/s41586020-2603-3 (2019).

104. Winkel, P. et al. Nondegenerate parametric amplifiers based on dispersion engineered Josephson-junction arrays. Phys. Rev. Appl. 13, 024015 (2020).

105. Winkel, P. et al. Implementation of a transmon qubit using superconducting granular aluminum. Phys. Rev. X 10, 031032 (2020).

106. Rigetti, C. T. Quantum Gates for Superconducting Qubits. PhD thesis, Yale Univ. (2009).

107. Lecocq, F. et al. Junction fabrication by shadow evaporation without a suspended bridge. Nanotechnology 22, 315302 (2011).

108. Pop, I. M. et al. Fabrication of stable and reproducible submicron tunnel junctions. J. Vacuum Sci. Technol. B 30, 010607 (2012)

109. Ambegaokar, V. \& Baratoff, A. Tunneling between superconductors. Phys. Rev. Lett. 11, 104-104 (1963).

110. Bergeal, N. et al. Phase-preserving amplification near the quantum limit with a Josephson ring modulator. Nature 465, 64-68 (2010).

111. Abdo, B., Kamal, A. \& Devoret, M. Nondegenerate three-wave mixing with the Josephson ring modulator. Phys. Rev. B 87, 014508 (2013).

112. Roy, A. \& Devoret, M. H. Introduction to parametric amplification of quantum signals with Josephson circuits. Comptes Rendus Phys. 17, 740-755 (2016).

113. Reagor, M. J. Superconducting Cavities for Circuit Quantum Electrodynamics. PhD thesis, Yale Univ. (2016)

114. Gambetta, J. et al. Qubit-photon interactions in a cavity: measurement-induced dephasing and number splitting. Phys. Rev. A 74, 042318 (2006)

115. Gambetta, J. et al. Quantum trajectory approach to circuit QED: quantum jumps and the Zeno effect. Phys. Rev. A 77, 012112 (2008).

116. Gambetta, J. M., Houck, A. A. \& Blais, A. Superconducting qubit with Purcell protection and tunable coupling. Phys. Rev. Lett. 106, 030502 (2011).

117. Srinivasan, S. J., Hoffman, A. J., Gambetta, J. M. \& Houck, A. A. Tunable coupling in circuit quantum electrodynamics using a superconducting charge qubit with a V-shaped energy level diagram. Phys. Rev. Lett. 106, 083601 (2011).
118. Diniz, I., Dumur, E., Buisson, O. \& Auffèves, A. Ultrafast quantum nondemolition measurements based on a diamond-shaped artificial atom. Phys. Rev. A 87 033837 (2013).

119. Dumur, É. et al. V-shaped superconducting artificial atom based on two inductively coupled transmons. Phys. Rev. B 92, 020515 (2015).

120. Zhang, G., Liu, Y., Raftery, J. J. \& Houck, A. A. Suppression of photon shot noise dephasing in a tunable coupling superconducting qubit. npj Quantum Inf. 3, 1 (2017).

121. Roy, T. et al. Implementation of pairwise longitudinal coupling in a three-qubit superconducting circuit. Phys. Rev. Appl. 7, 054025 (2017).

122. Devoret, M., Girvin, S. \& Schoelkopf, R. Circuit-QED: how strong can the coupling between a Josephson junction atom and a transmission line resonator be? Ann. Phys. 16, 767-779 (2007).

123. Houck, A. A. et al. Controlling the spontaneous emission of a superconducting transmon qubit. Phys. Rev. Lett. 101, 080502 (2008).

\section{ACKNOWLEDGEMENTS}

We thank S.M. Girvin, R.J. Schoelkopf, S. Nigg, H. Paik, A. Blais, H.E. Türeci, F. Solgun, V. Sivak, S. Touzard, N. Frattini, S. Shankar, C. Axline, V.V. Albert, K. Chou, A. Petrescu, D. Cody, A. Eickbusch, and E. Flurin for valuable discussions and the I. Siddiqi and B. Huard groups for using the early versions of PYEPR. This research was supported by the US Army Research Office (ARO) Grant No. W911NF-18-1-0212. Z.M. acknowledges partial support from the ARO (W911NF-16-1-0349). M.H.D. acknowledges partial support from the ARO (W911NF-18-1-0020) and the Air Force Office of Scientific Research (FA9550-19-1-0399). The view and conclusions contained in this document are those of the authors and should not be interpreted as representing the official policies, either expressed or implied, of the Army Research Office or the US Government. The US Government is authorized to reproduce and distribute reprints for Government purposes notwithstanding any copyright notation herein.

\section{AUTHOR CONTRIBUTIONS}

Z.K.M. conceived and developed the method and the theory, performed the experiments, and analyzed the data. Z.L. and M.H.D. contributed to the theory. I.M.P. and S.O.M. contributed to the measurement and fabrication of the devices L.C. assisted with the simulations. Z.K.M. and M.H.D. wrote the manuscript. All authors provided suggestions, discussed the results, and contributed to the manuscript.

\section{COMPETING INTERESTS}

The authors declare no competing interests.

\section{ADDITIONAL INFORMATION}

Supplementary information The online version contains supplementary material available at https://doi.org/10.1038/s41534-021-00461-8.

Correspondence and requests for materials should be addressed to Z.K.M.

Reprints and permission information is available at http://www.nature.com/ reprints

Publisher's note Springer Nature remains neutral with regard to jurisdictional claims in published maps and institutional affiliations.

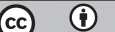

Open Access This article is licensed under a Creative Commons Attribution 4.0 International License, which permits use, sharing, adaptation, distribution and reproduction in any medium or format, as long as you give appropriate credit to the original author(s) and the source, provide a link to the Creative Commons license, and indicate if changes were made. The images or other third party material in this article are included in the article's Creative Commons license, unless indicated otherwise in a credit line to the material. If material is not included in the article's Creative Commons license and your intended use is not permitted by statutory regulation or exceeds the permitted use, you will need to obtain permission directly from the copyright holder. To view a copy of this license, visit http://creativecommons. org/licenses/by/4.0/.

(c) The Author(s) 2021 